\title{
Listing of Regional and State Political Science Associations
}

*These associations did not respond to our survey, and information is repeated from the March 1995 PS.

Associations

Officers

Publications

Meetings

\section{REGIONAL}

MIDWEST POLITICAL SCIENCE ASSOCIATION 6525 N. Sheridan Road, Chicago, IL 60626

(800) $782-1178$

Fax: (773) 508-3102

E-mail: jpeliss@luc.edu

Web Site: http://www.luc.edu/ orgs/mpsa
President: Arlene Saxonhouse, University of Michigan
President-elect: Harold Spaeth,
American Joumal of Political Science, Kenneth Meier, University of Wisconsin-Milwaukee, Editor Michigan State University

Executive Director: John Pelissero, Loyola University Chicago
Annual Convention Palmer House, Chicago 1997: April 10-12 1998: April 23-25 1999: April 15-17
NATIONAL CAPITAL

AREA POLITICAL

SCIENCE ASSOCIATION

(Serving: Delaware, District of

Columbia, Maryland,

Pennsylvania, Virginia, West

Virginia)

1319 18th St., NW,

Washington, DC 20036

(202) 296-6267
President: Joseph Cooper,

Johns Hopkins University

1st Vice President: Paul Herrnson,

University of Maryland

2nd Vice President: Lee Sigelman,

George Washington University

Secretary/Treasurer: Walter E. Beach,

Helen Dwight Reid Educational

Foundation

NEW ENGLAND
POLITICAL SCIENCE
ASSOCIATION

President: Barbara Craig,

Wesleyan University

President-elect: Gary Jacobsohn,

Williams College

Vice President: Maureen Moakley,

University of Rhode Island

Program Chair: Kathleen Moore, University of Connecticut
NORTHEASTERN

POLITICAL SCIENCE

ASSOCIATION
President: Nancy E. McGlen, Niagara University

Vice President \& Program Chair:

Thomas Brogran, Albright College

Executive Director: Joseph Melusky, Saint

Francis College

Treasurer: Donald Buzinkai, King's College
CAPSULE (newsletter), Morris Levitt, Spring Conference Howard Univesity, Editor Mount Vernon College Washington, DC March 22, 1997
President: Joanne Aviel, San Francisco State University

Vice-President/Program Chair: Larry Pippin, University of the Pacific

Secretary-Treasurer: Gerald McDaniel,
Polity, M.J. Peterson, University of Massachusetts, Editor
Annual Meeting

May 2-3, 1997

Radisson Hotel

New London, CT
POLITICAL SCIENCE

ASSOCIATION
Annual Meeting

May 10, 1997 


\begin{tabular}{|c|c|c|c|}
\hline Associations & Officers & Publications & Meetings \\
\hline $\begin{array}{l}\text { PACIFIC NORTHWEST } \\
\text { POLITICAL SCIENCE } \\
\text { ASSOCIATION } \\
\text { c/o Robbie Roy } \\
\text { Department of Political } \\
\text { Science, Campus Box } 12 \\
\text { Lewis \& Clark College } \\
\text { Portland, OR 97219 } \\
\text { Phone: (503) 768-7755 } \\
\text { E-mail: roy@clark.edu } \\
\text { Web Site: http://www.orst.edu/ } \\
\text { dept/pol_sci/pnwpsa }\end{array}$ & $\begin{array}{l}\text { President, 1996-97: Curtis Johnson, Lewis \& } \\
\text { Clark College } \\
\text { President-elect, 1997-98: Dick Olufs, Pacific } \\
\text { Lutheran University } \\
\text { Executive Secretary-Treasurer: Robbie Roy, } \\
\quad \text { Lewis and Clark College } \\
\text { Ex Officio: Eugene Hogan, Western } \\
\text { Washington University }\end{array}$ & & $\begin{array}{l}\text { Annual Meeting } \\
\text { The Resort at Coeur } \\
\text { d'Alene, Idaho }\end{array}$ \\
\hline $\begin{array}{l}\text { SOUTHERN POLITICAL } \\
\text { SCIENCE ASSOCIATION } \\
\text { Department of Political } \\
\text { Science, University of } \\
\text { Mississippi, University, } \\
\text { MS 38655 } \\
\text { Phone: (601) 232-5673 } \\
\text { E-mail: spsa@cypress. } \\
\text { mcsr.olemiss.edu } \\
\text { Web Site: http://www. } \\
\text { olemiss.edu/orgs/spsa }\end{array}$ & $\begin{array}{l}\text { President: Earl Black, Rice University } \\
\text { President-elect: Ronald E. Weber, } \\
\text { University of Wisconsin-Milwaukee } \\
\text { Vice President and Program Chair: } \\
\text { Karen O'Connor, American University } \\
\text { Recording Secretary: Walter Beach, } \\
\text { Helen Dwight Reid Foundation } \\
\text { Executive Director: Gregory S. Mahler, } \\
\text { University of Mississippi }\end{array}$ & $\begin{array}{l}\text { The Joumal of Politics, David Lowery, } \\
\text { University of North Carolina- } \\
\text { Chapel Hill, Editor }\end{array}$ & $\begin{array}{l}\text { Annual Meeting } \\
\text { Omni Waterside Hotel } \\
\text { Norfolk, Virginia } \\
\text { November 5-8, } 1997\end{array}$ \\
\hline $\begin{array}{l}\text { SOUTHWESTERN } \\
\text { POLITICAL SCIENCE } \\
\text { ASSOCIATION }\end{array}$ & $\begin{array}{l}\text { President: Patricia Hurley, Texas } \\
\text { A\&M University } \\
\text { President-elect: Robert Stein, Rice University } \\
\text { Vice President and Program Chair: } \\
\text { Cynthia Opheim, Southwest Texas State } \\
\text { University } \\
\text { Secretary-Treasurer: Harold F. Bass, Jr., } \\
\text { Ouachita Baptist University }\end{array}$ & $\begin{array}{l}\text { Social Science Quarterly (cooperatively } \\
\text { with the Southwestern Social Science } \\
\text { Association), Robert L. Lineberry, } \\
\text { University of Houston, Editor }\end{array}$ & $\begin{array}{l}\text { Annual Meeting } \\
\text { March 26-29, } 1997 \\
\text { Fairmont Hotel } \\
\text { New Orleans, LA }\end{array}$ \\
\hline $\begin{array}{l}\text { WESTERN POLITICAL } \\
\text { SCIENCE ASSOCIATION }\end{array}$ & $\begin{array}{l}\text { President: Jane Bayes, California State } \\
\text { University-Northridge } \\
\text { Program Chair: Luis Fraga, Stanford } \\
\text { University } \\
\text { Secretary-Treasurer: Elizabeth Moulds, } \\
\text { California State University, Sacramento }\end{array}$ & $\begin{array}{l}\text { Political Research Quarterly, Walter J. } \\
\text { Stone, University of Colorado, } \\
\text { Boulder, Editor }\end{array}$ & $\begin{array}{l}\text { Annual Meeting } \\
\text { March 13-15, } 1997 \\
\text { Tucson, AZ } \\
\text { March 20-22, } 1998 \\
\text { Los Angeles, CA }\end{array}$ \\
\hline
\end{tabular}

\section{STATE}

ALABAMA POLITICAL SCIENCE ASSOCIATION

President: D'Linnell Finley,

University of Alabama

Past President: Digambar Mishra,

Miles College

Vice President: James B. Allen, Jr.,

Jacksonville State University

Secretary-Treasurer: Jerry Gilbert,

Jacksonville State University

ALASKA POLITICAL

SCIENCE GROUP

(informal network)

ARKANSAS POLITICAL

SCIENCE ASSOCIATION

Web Site: http://www.

csm. astate.edu/ $\sim$ posc/polisci/ arkpsa.html
Contact Person: Clive S. Thomas,

University of Alaska-Juneau
President: W. Martin James, Henderson State University

Vice President: Douglas L. Reed, Ouachita Baptist University

Secretary-Treasurer: C. Jeddy Lever, Henderson State University

Program Chair: Jeffrey J. Ryan,

University of Arkansas
Directory of Alabama Political Scientists
Annual Meeting April 11-12, 1997 Jacksonville State University 


\begin{tabular}{|c|c|c|c|}
\hline Associations & Officers & Publications & Meetings \\
\hline $\begin{array}{l}\text { FLORIDA POLITICAL } \\
\text { SCIENCE ASSOCIATION }\end{array}$ & $\begin{array}{l}\text { President: Renu Khator, University of } \\
\text { South Florida } \\
\text { 1st Vice President: Susan MacManus, } \\
\text { University of South Florida } \\
\text { 2nd Vice President: Ed Schwerin, } \\
\text { Florida Atlantic University } \\
\text { Secretary-Treasurer: Gary L. Maris, } \\
\text { Stetson University }\end{array}$ & $\begin{array}{l}\text { The Political Chronicle } \\
\text { Hud Reynolds, St. Leo College, } \\
\text { Editor }\end{array}$ & $\begin{array}{l}\text { Annual Meeting } \\
\text { April 4-5, } 1997 \\
\text { Ft. Lauderdale, FL }\end{array}$ \\
\hline $\begin{array}{l}\text { GEORGIA POLITICAL } \\
\text { SCIENCE ASSOCIATION }\end{array}$ & $\begin{array}{l}\text { President: Willoughby Jarrell, Kennesaw } \\
\text { State College } \\
\text { Vice President and Program Chair: Jan } \\
\text { Mabie, Georgia College } \\
\text { Secretary-Treasurer: Brian Murphy, North } \\
\text { Georgia College }\end{array}$ & $\begin{array}{l}\text { The Southeastern Political Review, } \\
\text { Roger Pajari, Georgia Southern } \\
\text { University, Editor }\end{array}$ & Annual Meeting \\
\hline $\begin{array}{l}\text { *IDAHO POLITICAL } \\
\text { SCIENCE ASSOCIATION }\end{array}$ & $\begin{array}{l}\text { President: Richard Henry Foster, Idaho } \\
\text { State University } \\
\text { Secretary-Treasurer: William Skillern, Boise } \\
\text { State University }\end{array}$ & $\begin{array}{l}\text { Idaho Joumal of Politics, Idaho State } \\
\text { University }\end{array}$ & \\
\hline $\begin{array}{l}\text { ILLINOIS POLITICAL } \\
\text { SCIENCE ASSOCIATION }\end{array}$ & $\begin{array}{l}\text { President: Robert Bradley, Illinois State } \\
\text { University } \\
\text { Secretary-Treasurer: Tari Renner, Illinois } \\
\text { Wesleyan University }\end{array}$ & $\begin{array}{l}\text { The Illinois Political Science Review } \\
\text { Jim Simeone, Illinois Wesleyan } \\
\text { University, Editor } \\
\text { IPSA Newsletter Peter Ruckman, } \\
\text { Northern Illinois University, Editor }\end{array}$ & $\begin{array}{l}\text { Annual Meeting } \\
\text { October, } 1997\end{array}$ \\
\hline $\begin{array}{l}\text { INDIANA POLITICAL } \\
\text { SCIENCE ASSOCIATION }\end{array}$ & $\begin{array}{l}\text { President and Program Chair: Margaret } \\
\text { Brabant, Butler University } \\
\text { Vice President: Maurice Eisenstein, Purdue } \\
\text { University-Calumet } \\
\text { Treasurer and Recorder: Melinda Rider, } \\
\text { IUPUI }\end{array}$ & $\begin{array}{l}\text { Journal Editor: Fred Meyer, Ball State } \\
\text { University }\end{array}$ & $\begin{array}{l}\text { Annual Meeting } \\
\text { April or May } 1997\end{array}$ \\
\hline $\begin{array}{l}\text { IOWA CONFERENCE OF } \\
\text { POLITICAL SCIENTISTS } \\
\text { c/o Don Racheter } \\
\text { Dept. of Political Science } \\
\text { Central College } \\
\text { Pella, IA 50219 } \\
\text { Phone: (515) 628-5259 } \\
\text { E-mail: racheterd@central.edu }\end{array}$ & $\begin{array}{l}\text { President: Eduardo Magalhaes, III, Simpson } \\
\text { College } \\
\text { Vice President: Paul Gardner, Luther College } \\
\text { Executive Secretary: Don Racheter, Central } \\
\text { College }\end{array}$ & & $\begin{array}{l}\text { Annual Meeting } \\
\text { Fall 1997-TBA } \\
\text { Luther College } \\
\text { Decorah, IA }\end{array}$ \\
\hline $\begin{array}{l}\text { KANSAS POLITICAL } \\
\text { SCIENCE ASSOCIATION }\end{array}$ & $\begin{array}{l}\text { Contact: Ronald A. Francisco, University } \\
\text { of Kansas }\end{array}$ & & Mid to Late Fall \\
\hline $\begin{array}{l}\text { KENTUCKY POLITICAL } \\
\text { SCIENCE ASSOCIATION }\end{array}$ & $\begin{array}{l}\text { President: Edward M. Yager, Bowling } \\
\text { Green University }\end{array}$ & $\begin{array}{l}\text { Proceedings of the Annual Meeting } \\
\text { (includes a directory of Kentucky } \\
\text { political scientists) }\end{array}$ & $\begin{array}{l}\text { Regularly, first Friday \& } \\
\text { Saturday in March at site } \\
\text { of President's institution }\end{array}$ \\
\hline $\begin{array}{l}\text { LOUISIANA POLITICAL } \\
\text { SCIENCE ASSOCIATION }\end{array}$ & $\begin{array}{l}\text { President: Kurt Corbello, Southeastern } \\
\text { Louisiana University } \\
\text { Vice President \& Program Chair: } \\
\text { Judith Haydel, McNeese State University } \\
\text { Secretary-Treasurer: Donn Kurtz II, } \\
\text { University of Southwestern Louisiana }\end{array}$ & & $\begin{array}{l}\text { Annual Meeting } \\
\text { March }\end{array}$ \\
\hline $\begin{array}{l}\text { MICHIGAN CONFERENCE } \\
\text { OF POLITICAL SCIENTISTS }\end{array}$ & $\begin{array}{l}\text { President: Richard Elling, Wayne State } \\
\text { University } \\
\text { President-elect: Elaine Martin, Eastern } \\
\text { Michigan University } \\
\text { Secretary: Mary Young, Southwestern } \\
\text { Michigan College }\end{array}$ & $\begin{array}{l}\text { Michigan Political Science Directory, } \\
\text { Ellis Perlman, University of } \\
\text { Michigan-Flint }\end{array}$ & $\begin{array}{l}\text { Annual Meeting } \\
\text { October } 1997\end{array}$ \\
\hline $\begin{array}{l}\text { MINNESOTA POLITICAL } \\
\text { SCIENCE ASSOCIATION }\end{array}$ & $\begin{array}{l}\text { President: William Flannigan, University of } \\
\text { Minnesota College } \\
\text { Secretary-Treasurer: Orville Schmidt, St. } \\
\quad \text { Cloud State University }\end{array}$ & $\begin{array}{l}\text { Director of Minnesota Political Scientists } \\
\text { Perspectives on Minnesota } \\
\quad \text { Government and Politics (Gieske) }\end{array}$ & $\begin{array}{l}\text { Annual Meeting } \\
\text { Fall }\end{array}$ \\
\hline
\end{tabular}




\begin{tabular}{|c|c|c|c|}
\hline Associations & Officers & Publications & Meetings \\
\hline $\begin{array}{l}\text { MISSISSIPPI POLITICAL } \\
\text { SCIENCE ASSOCIATION }\end{array}$ & $\begin{array}{l}\text { President: Stephen Rozman, University } \\
\text { of Southern Mississippi } \\
\text { Vice President: Ellen Singh, } \\
\text { Mississippi Valley State University } \\
\text { Secretary-Treasurer: David Breaux, } \\
\text { Tougaloo College }\end{array}$ & & $\begin{array}{l}\text { Annual Meeting } \\
\text { February }\end{array}$ \\
\hline $\begin{array}{l}\text { NEBRASKA POLITICAL } \\
\text { SCIENCE ASSOCIATION }\end{array}$ & $\begin{array}{l}\text { Contact: Charles H. Evans, Hastings } \\
\text { College }\end{array}$ & & \\
\hline
\end{tabular}

*NEW JERSEY POLITICAL President: David P. Rebovich, Rider College Annual Meeting

SCIENCE ASSOCIATION

\begin{tabular}{lll}
\hline NEW YORK STATE & President: Victoria M. Kraft, Ithaca College & Annual Meeting \\
POLITICAL SCIENCE & Vice President and Program Chair: & .
\end{tabular}

POLITICAL SCIENCE ASSOCIATION

Jeffrey Kraus, Wagner College

Secretary-Treasurer: Arthur Galub, Bronx

Community College

\begin{tabular}{lll}
\hline NORTH CAROLINA & President: Ruth Ann Strickland, Appalachian & Southeastern Political Review \\
POLITICAL SCIENCE & State University & Annual Meeting \\
ASSOCIATION & Vice President: Rudy Zarzar, Elon College & March 21-22, 1997 \\
& Treasurer: Cynthia Combs, University of & \\
& North Carolina-Charlotte & \\
& Secretary: James Daniel Steele, North & \\
& Carolina A\&T State University & \\
\hline
\end{tabular}

OHIO ASSOCIATION OF
ECONOMISTS AND
POLITICAL SCIENTISTS

President: Craig Ramsay (POL), Ohio

Wesleyan University

Vice President: Barbara Rolleston (ECON), Baldwin-Wallace College

Treasurer: Robert Ebert (ECON), Baldwin-Wallace College

Secretary: William Binning (POL), Youngstown State University

OKLAHOMA POLITICAL SCIENCE ASSOCIATION
President: Anthony Brown, University Center at Tulsa

President-Elect: John Ulrich, East Central University

Executive Director: Bill Parle, Oklahoma State University
Ohio Journal of Economics and Politics, James Sperling, University of Akron, Political Science Editor; Henry Rennie, Heidelberg College, Economics Editor; and Ernest Brass III, Managing Editor

Newsletter: James Jordan (POL), Ohio University-Zanesville

Directory of Oklahoma Political Science Programs and Individual Members 1995

Oklahoma Politics, Robert Darcy, Oklahoma State University, and Greg Scott, University of Central Oklahoma, Editors
Annual Meeting

October 17-18, 1997 Baldwin-Wallace College Berea, $\mathrm{OH}$
Annual Meeting November Ada, OK
Commonwealth: A Journal of Political Science, Thomas J. Baldino, Wilkes University, Editor Pennsylvania

Political Scientists Newsletter, Craig Wheeland, Villanova University, Editor
President: Joseph Melusky, St. Francis Co
First Vice President: Kathleen McQuaid, Mansfield University

Second Vice President: Craig M. Wheeland, Villanova University

Secretary: Thomas C. Brogan, Albright College

Treasurer: Donald Buzinkai, King's College
*SOUTH CAROLINA POLITICAL SCIENCE ASSOCIATION

\section{President: Laurence W. Moreland,}

The Citadel

Vice-President and Program Chair:

Donald J. Puchala, University of South

Carolina

Secretary-Treasurer: Eileen Kirley-Tallon,

Francis Marion University 


\begin{tabular}{|c|c|c|c|}
\hline Associations & Officers & Publications & Meetings \\
\hline $\begin{array}{l}\text { TENNESSEE POLITICAL } \\
\text { SCIENCE ASSOCIATION }\end{array}$ & $\begin{array}{l}\text { President: Dwight Tays, David Lipscomb } \\
\text { University } \\
\text { President-elect: Sharon Whitney, } \\
\text { Tennessee Technological University } \\
\text { Vice President: Ken Holland, University } \\
\text { of Memphis } \\
\text { Secretary: Larry Hall, Belmont University } \\
\text { Treasurer: Mark Byrnes, Middle Tennessee } \\
\text { State University }\end{array}$ & $\begin{array}{l}\text { TPSA Newsletter: David Kanervo, } \\
\text { Austin Peay State University, } \\
\text { Editor }\end{array}$ & $\begin{array}{l}\text { Annual Meeting } \\
\text { April 11-12, } 1997 \\
\text { University of Memphis }\end{array}$ \\
\hline $\begin{array}{l}\text { VIRGINIA CONFERENCE } \\
\text { OF POLITICAL SCIENTISTS }\end{array}$ & Contact: Edward Lynch, Hollins College & & $\begin{array}{l}\text { Annual Meeting } \\
\text { December }\end{array}$ \\
\hline $\begin{array}{l}\text { WEST VIRGINIA } \\
\text { POLITICAL SCIENCE } \\
\text { ASSOCIATION }\end{array}$ & $\begin{array}{l}\text { President: Joseph McCoy, Marshall University } \\
\text { Vice President and Program Chair: Richard } \\
\text { Brisbin, West Virginia University } \\
\text { Secretary: James Forrester, West Liberty } \\
\text { State College } \\
\text { Treasurer: Rodney Pyles, Assessor, } \\
\text { Monongalia County }\end{array}$ & $\begin{array}{l}\text { Southeastern Political } \\
\text { Review (in cooperation with Georgia } \\
\text { Political Science Association) }\end{array}$ & $\begin{array}{l}\text { Annual Meeting } \\
\text { October } 17-18 \\
\text { West Virginia University } \\
\text { Morgantown, WV }\end{array}$ \\
\hline $\begin{array}{l}\text { WISCONSIN POLITICAL } \\
\text { SCIENCE ASSOCIATION }\end{array}$ & $\begin{array}{l}\text { President: John Oh, University of Wisconsin- } \\
\text { Whitewater } \\
\text { Vice President: Herbert Kritzer, University } \\
\text { of Wisconsin-Madison } \\
\text { Treasurer: Cecilia Manrique, University } \\
\text { of Wisconsin-La Crosse } \\
\text { Secretary: Richard Christofferson, University } \\
\text { of Wisconsin-Stevens Points }\end{array}$ & $\begin{array}{l}\text { Wisconsin Political Scientist, Morton } \\
\text { Sipress, University of Wisconsin- } \\
\text { Eau Claire, Editor } \\
\text { Directory of Wisconsin Political } \\
\text { Scientists, compiled by the } \\
\text { Association president }\end{array}$ & $\begin{array}{l}\text { Annual Meeting } \\
\text { October }\end{array}$ \\
\hline
\end{tabular}

\section{President of the Political Science Association of Nepal?}

Check out APSANet, the American Political Science Association Online, for the latest contact information on national political science associations, state and regional associations, area studies groups, and much more. Visit APSANet at:

\section{http://www.apsanet.org}

